Knie J. $2020 \cdot 2$ (Suppl 1):S10-S19

https://doi.org/10.1007/s43205-020-00062-z

Online publiziert: 14. Mai 2020

(c) Springer Medizin Verlag GmbH, ein Teil von Springer Nature 2020

\section{Einleitung}

Die SARS-CoV-2(„Severe Acute Respiratory Syndrome Corona Virus 2")-Pandemie hat die Orthopädie und Unfallchirurgie in vielen Ländern hart getroffen $[28,34]$. Das neuartige Coronavirus hat zu einer weltweiten Pandemie geführt, die die Gesundheitssysteme einzelner Regionen an die Grenzen ihrer Kapazität geführt hat.

Um eine exponentielle Verbreitung von SARS-CoV-2 zu verhindern, waren verschiedene Schutzmaßnahmen notwendig, die eine Tröpfcheninfektion, aber auch eine Übertragung durch kontaminierte Oberflächen verhindern. $\mathrm{Zu}$ den Schutzmaßnahmen zur Eindämmung der Pandemie gehören die Abstandsregeln (1,5 m), die Kontaktbeschränkung und das Tragen von Masken [34]. Diese Maßnahmen beeinträchtigen den klinischen Alltag in der Orthopädie und Unfallchirurgie erheblich [28]. Gerade weil die Übertragung von SARSCoV-2 hauptsächlich über Tröpfchen erfolgt, hat sich das klinische Interesse an kontaktloser Diagnostik in den letzten Wochen verstärkt. Dabei treten insbesondere Methoden der Telemedizin in den Vordergrund, die bisher im dicht besiedelten Deutschland nur geringe Relevanz hatten (bis auf die klassische Beratung am Telefon) [16, 31, 36]. Bisher hatten diese Verfahren eher in dünn besiedelten Ländern wie Norwegen, Kanada oder Australien oder in der Militärmedizin eine Bedeutung [7-9, 35, 36]. Dabei werden Arzt-zu-Arzt- und

Luisa Backhaus · Sebastian Bierke · Katrin Karpinski · Martin Häner · Wolf Petersen

Sportklinik Berlin und Klinik für Orthopädie und Unfallchirurgie, Martin Luther Krankenhaus, Berlin Grunewald, Berlin, Deutschland

\title{
SARS-CoV-2-Pandemie und ihre Auswirkungen auf Orthopädie und Unfallchirurgie: „Booster" für die Telemedizin
}

Arzt-zu-Patient-Konsultationen unterschieden (• Abb. 1). Im Rahmen der COVID-19-Krise besteht eher Interesse an Arzt-zu-Patient-Konsultationen, um die Kontaktsperren zu umgehen.

Technisch werden computergestützte Telemedizinanwendungen mit E-MailKonversationen von Videokonferenzsystemen unterschieden. Für die Arztzu-Patient-Konsultation sind Videokonferenzsysteme vorteilhaft, da die klassische Anamnese durch die zusätzliche Bildübertragung um die Inspektion und einfache Untersuchungen ergänzt werden kann. Bei den Arzt-PatientKonsultationen werden außerdem Anwendungen zur Primärdiagnostik von Anwendungen zur Überwachung von Therapieverfahren (z.B. postoperative Rehabilitation) unterschieden.

Obwohl einer virtuellen orthopädischen Untersuchung möglicherweise die wesentlichen Elemente der Palpation und die Möglichkeit dynamischer Tests fehlen, erfordert die aktuelle Situation, dass wir uns mit den verfügbaren telemedizinischen Ressourcen beschäftigen. Erste Verfahren zur inspektorischen Erfassung der Gelenkbeweglichkeit und Selbstpalpation des Gelenkes durch den Patienten liegen vor. Interessante neue Möglichkeiten zur virtuellen körperlichen Untersuchung eröffnen sich durch den Gebrauch mittlerweile kostengünstiger Sensoren. Diese eignen sich insbesondere für die Überwachung von Therapiemaßnahmen und der postoperativen Rehabilitation.

Die Chance dieser Krise liegt sicher auch darin, die Möglichkeiten der Tele- medizin für die Orthopädie und Unfallchirurgie zu nutzen und weiter zu verbessern. In den Zeiten nach der Krise werden diese Verfahren sicher neue Möglichkeiten in der internationalen Patientenkommunikation eröffnen

Mit diesem Übersichtsartikel möchten wir die heutigen Möglichkeiten und Grenzen der Telemedizin für die orthopädische Primärdiagnostik und Rehabilitationskontrolle vorstellen und diskutieren. Dabei soll dieses Thema einerseits unter praktischen Gesichtspunkten, andererseits aber auch unter wissenschaftlichen Aspekten beleuchtet werden.

\section{Systeme für eine Videosprech- stunde}

Es werden verschiedene Systeme für die Durchführung von Videosprechstunden angeboten (•Tab. 1). Die meisten Systeme erlauben eine „Online“-Terminvereinbarung und die Möglichkeit, dass sich Arzt und Patient im Rahmen der virtuellen Sprechstunde sehen (• Abb. 2 und 3).

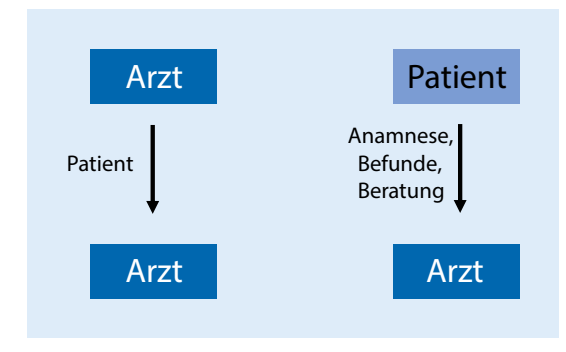

Abb. 1 A Möglichkeiten der telemedizinischen Interaktion 
Tab. 1 Übersicht über verschiedene Systeme für Videosprechstunden (Auswahl [15])

\begin{tabular}{|c|c|c|c|c|c|}
\hline System & Hersteller & $\begin{array}{l}\text { Direkte Kom- } \\
\text { munikation } \\
\text { Arzt-Patient }\end{array}$ & Gruppen & Lizenzen (Preise zzgl. MwSt.) & $\begin{array}{l}\text { Installation bzw. } \\
\text { PVS notwendig }\end{array}$ \\
\hline $\begin{array}{l}\text { arztkonsultation.de } \\
\text { www.arztkonsultation.de }\end{array}$ & $\begin{array}{l}\text { arztkonsultation } \\
\text { ak GmbH }\end{array}$ & Ja & $\begin{array}{l}\text { Ja, bis zu } \\
3 \text { Personen }\end{array}$ & $\begin{array}{l}\text { Kostenlos während der Krise, danach } \\
49 € / \text { Monat }\end{array}$ & Nein \\
\hline $\begin{array}{l}\text { Doctolib } \\
\text { www.doctolib.de }\end{array}$ & Doctolib GmbH & Ja & Nein & $\begin{array}{l}\text { Kostenlose Videosprechstunde während } \\
\text { der COVID-19-Pandemie }\end{array}$ & Nein \\
\hline $\begin{array}{l}\text { jameda } \\
\text { www.jameda.de }\end{array}$ & Jameda GmbH & Ja & k. A. & $\begin{array}{l}\text { Ab sofort } 6 \text { Monate kostenlos für Allge- } \\
\text { meinmediziner, Hausärzte und Internis- } \\
\text { ten }\end{array}$ & k. A. \\
\hline $\begin{array}{l}\text { samedi } \\
\text { www.samedi.de }\end{array}$ & Samedi GmbH & $\mathrm{Ja}$ & k. A. & $\begin{array}{l}\text { Für Neukunden bis } 30.06 .2020 \text { kostenlos, } \\
\text { anschließend } 65 € / \text { Monat }\end{array}$ & Nein \\
\hline $\begin{array}{l}\text { TeleKonsil } \\
\text { www.tele-konsil.de }\end{array}$ & Snapview GmbH & Ja & $\begin{array}{l}\text { Bis zu } 5 \text { Per- } \\
\text { sonen }\end{array}$ & $\begin{array}{l}\text { Während Covid-19 } 29 € \text { für } 6 \text { Monate, } \\
\text { danach } 59 € / \text { Monat }\end{array}$ & $\begin{array}{l}\text { Keine Installation } \\
\text { bzw. PVS notwen- } \\
\text { dig, optionale An- } \\
\text { bindung an TeleArzt } \\
\text { möglich }\end{array}$ \\
\hline $\begin{array}{l}\text { Tomedo } \\
\text { www.tomedo.de }\end{array}$ & Zollsoft & $\mathrm{Ja}$ & Nein & 3,99/Sprechstunde & Nein \\
\hline
\end{tabular}

Es existieren Systeme, bei denen die Fremdbefunde (z.B. Radiologie) vorher direkt in das System hochgeladen werden können. Hier erscheint die Möglichkeit der Bildschirmteilung sinnvoll, da so radiologische Aufnahmen zusammen mit dem Patienten betrachtet werden können. Auch die Dokumentation der Konsultation (Arztbrief) kann in einigen Videosprechstundensystemen gespeichert werden.

$\mathrm{Da}$ in Krankenhäusern meist ein Krankenhausinformationssystem (KIS) oder in Praxen ein etabliertes Praxisprogramm existiert, kann es sinnvoll sein, hier zweigleisig zu verfahren (• Abb. 2 und 3). Dabei werden abrechnungsrelevante Patienteninformationen, Checklisten und Radiologiebefunde vor der Konsultation in das KIS übertragen. Die Dokumentation der Inhalte der Konsultation (Arztbrief) erfolgt ebenfalls über das KIS und wird auch dort gespeichert.

\section{Vorbereitung auf eine Videosprechstunde}

Im Vorfeld ist es sinnvoll, dem Patienten eine Checkliste mit Hinweisen zur Durchführung der Konsultation zukommen zu lassen (•Tab. 2). Darin sollten Hinweise zur Ausleuchtung und Kameraposition und Bekleidung gegeben werden. Eine kurze, möglichst eng anliegende Sporthose ist als Beinbekleidung vor- teilhaft. Auch ein Anamnesefragebogen kann im Vorfeld übermittelt werden, um die Effektivität der Sprechstunde zu erhöhen (•Tab. 3).

Von ärztlicher Seite sollte darauf geachtet werden, dass der Raum gut ausgeleuchtet ist und dass keine Gegenlichtsituation erzeugt wird (nicht mit dem Rücken zu einem Fenster oder einer Leuchte sitzen). Anatomische Modelle oder vorbereitete Bilder können als zusätzliche visuelle Hilfsmittel hilfreich sein (• Abb. 3).

\section{Virtuelle Knieuntersuchung}

Den Hauptbestandteil der Videosprechstunde macht das Gespräch aus, das eine Rekapitulation und Vertiefung der anhand des Fragebogens erstellten Anamnese beinhaltet.

Die Möglichkeit der Videoübertragung erlaubt jedoch die Anamnese um gewisse Aspekte der Inspektion und körperlichen Untersuchung zu erweitern. Eine zentrale Bedeutung besitzt die Inspektion mit Beurteilung von Haut, Erythem, Erguss und muskulärer Atrophie. Hier kommt es auf eine gute Bildqualität an (cave Ausleuchtung, Gegenlicht).

Zur Eigenpalpation des Kniegelenkes zur Angabe von Schmerzpunkten eignet sich die Quadrantenmethode (• Abb. 4). Dabei wird der Patient aufgefordert, die
Patella mit einem Stift zu markieren. Schmerzen im medial-kaudalen und lateral-kaudalen Quadranten sprechen für Meniskussymptome oder eine das Kompartiment betreffende Gonarthrose. Schmerzen in den kranialen Quadranten sind eher dem Femoropatellargelenk zuzuordnen.

Auch die Beweglichkeit kann videoassistiert evaluiert werden. Diese kann im Stehen und Sitzen erfasst werden. Praktisch ist die Beurteilung im Sitzen. Dabei sollte der Patient aufgefordert werden, seitlich auf dem Stuhl sitzend das Knie voll zu strecken und anschließend voll zu beugen ( $\bullet$ Abb. 5). Dabei soll der Patient die Ferse in sitzender Position zum Körper ziehen (• Abb. 5). Bei der Erfassung der Extension im Sitzen ist zu beachten, dass diese durch die flektierte Hüfte limitiert wird (Zug der gedehnten Beugemuskeln). Eine neutrale Extension $\left(0^{\circ}\right)$ kann im Sitzen ein Normalbefund sein. Im Stehen hingegen kann auch die Überstreckung erfasst werden, da in dieser Position die Beugemuskeln entspannt sind. Dabei wird der Patient aufgefordert, das Knie nach hinten zu drücken während der Fuß belastet bleibt, anschließend kann die aktive Flexion erfasst werden, indem der Patient den Fuß an das Gesäß führen soll (• Abb. 6).

Ein webbasiertes Goniometer (Protractor; Ben Burlingham [32]), das mit den meisten Anwendungen kompatibel 
Knie J. 2020 - 2 (Suppl 1):S10-S19 https://doi.org/10.1007/s43205-020-00062-z

(c) Springer Medizin Verlag GmbH, ein Teil von Springer Nature 2020

L. Backhaus · S. Bierke · K. Karpinski · M. Häner · W. Petersen

\section{SARS-CoV-2-Pandemie und ihre Auswirkungen auf Orthopädie und Unfallchirurgie: „Booster“ für die Telemedizin}

\section{Zusammenfassung}

Mit dem Ausbruch der COVID-19-Pandemie

ist das Interesse an telemedizinischen

Versorgungsmöglichkeiten gewachsen.

Diese betreffen einerseits den Bereich der

Diagnostik, aber auch die Überwachung von

Therapieverläufen und Rehabilitationsmaß-

nahmen.

Aufgrund der derzeitigen Ressourcenbeschränkungen sahen sich viele Orthopäden und Unfallchirurgen gezwungen, Videosprechstunden einzurichten, obwohl Standards für orthopädische Konsultationen bisher nur unzureichend entwickelt sind. Um die Effizienz der virtuellen Orthopädie zu maximieren, sollten die Patienten auf den virtuellen Besuch vorbereitet werden (Checkliste mit spezifischen Anweisungen zur Kamerapositionierung, Körperpositionierung,
Einstellung und Kleidung, Prüfung der audiovisuellen Fähigkeiten). Klassische diagnostische Maßnahmen wie Anamnese, Inspektion und Beurteilung radiologischer Befunde sind in der Videosprechstunde möglich. Es entfällt jedoch die Möglichkeit der funktionellen Untersuchung (Stabilität des Kniegelenkes, Schultertests). Auch wenn erste wissenschaftliche Studien gezeigt haben, dass die telemedizinische Diagnostik der konventionellen Diagnostik nicht unterlegen ist, so fehlen doch validierte Untersuchungsprotokolle und Methoden. Die postoperative Überwachung von Rehabilitationsmaßnahmen kann z. B. durch den Einsatz von Sensoren erleichtert werden. Mit moderner Sensorik ist mittlerweile eine kostengünstige Erfassung der Gelenkbeweglichkeit und Gelenkstellung möglich und wird bereits im Bereich der Rehabilitation nach Rekonstruktion des vorderen Kreuzbandes eingesetzt. Auch hier ist sicher weitere Forschung notwendig, um diese Methoden zu validieren.

Wir glauben, dass die derzeitige Pandemie Chancen bietet, die Möglichkeiten der Telemedizin für die Orthopädie und Unfallchirurgie auszubauen, um sie auch in der Zukunft weiter zu nutzen (z. B. bei der Versorgung von Patienten aus dem Ausland oder in dünn besiedelten Gebieten sowie der Betreuung von Hochleistungs- und Profisportlern).

Schlüsselwörter

Covid-19 · Orthopäden · Unfallchirurgen . Operationen · Untersuchung · Diagnostik . Validierung

\section{The SARS-CoV-2 pandemic and its impact on orthopedics and trauma surgery: a boost for telemedicine}

\section{Abstract}

With the outbreak of the COVID-19 pandemic, interest in telemedical care options has grown. Today's telemedicine options relate not only to the area of diagnostics, but also to the monitoring of therapy courses and rehabilitation measures. Due to the current resource constraints, many orthopedic surgeons and trauma surgeons have been forced to set up video consultations, although standards for orthopedic video consultations have so far been insufficiently developed. In order to maximize the efficiency of virtual orthopedics, patients should be prepared for the virtual visit (checklist with specific instructions on camera positioning, body positioning, adjustment and clothing, testing of audiovisual skills). Classic diagnostic measures such as anamnesis, inspection and assessment of radiological findings are possible in the video consultation. One major disadvantage is, that there is no possibility for a functional examination (stability of the knee joint, shoulder tests). Although initial controlled scientific studies have shown that telemedical diagnostics are not inferior to conventional diagnostics, there are still no validated examination protocols and methods. Postoperative monitoring of rehabilitation measures can, for example, be made easier by the use of sensors. With modern sensors, inexpensive detection of joint mobility and joint position is now possible and is already used in the area of rehabilitation after reconstruction of the anterior cruciate ligament. Here too, further research is certainly necessary to validate these methods. We believe that the current pandemic offers opportunities to further expand the possibilities of telemedicine for orthopedics and trauma surgery in order to continue to use it in the future (e.g. in the care of patients from abroad or in sparsely populated areas, care of professional athletes).

\section{Keywords}

Covid-19 - Orthopedic surgeon - Trauma surgeon · Surgery - Examination - Diagnostics . Validation ist, kann sinnvoll sein ([12]; • Abb. 5). Die Zuverlässigkeit von Bewegungsumfangsmessungen von Gelenken auf der Grundlage von Foto- und Videodokumentation konnte in mehreren Untersuchungen überzeugen $[4,12,23,38]$.

Ein möglicher Meniskustest ist der Thessaly-Test, bei dem der Patient auf dem betroffenen Bein steht und den Körper nach innen und außen dreht (- Abb. 6). Als positiver Befund gelten Schmerzen und mechanische Missemp- findungen über der Seite des betroffenen Meniskus [20].

Eine Möglichkeit der dynamischen Untersuchung sind einbeinige Kniebeugen [27]. Ein valgischer Kollaps kann in der einbeinigen Kniebeuge auf eine Dysbalance der Hüftabduktoren oder eine Fußdeformität (Pes planovalgus) hinweisen.

\section{Webgestützte Rehabilitation}

Telemedizinische Anwendungen sind jedoch nicht nur für Sprechstundenkonsultationen interessant. Auch die telemedizinische Steuerung und Überwachung von Rehabilitationsmaßnahmen besitzt angesichts der SARS-CoV-2-Pandemie auch in dicht besiedelten Ländern wie Deutschland Relevanz [5].

Dabei muss zwischen Systemen zur ärztlichen Überwachung von postopera- 


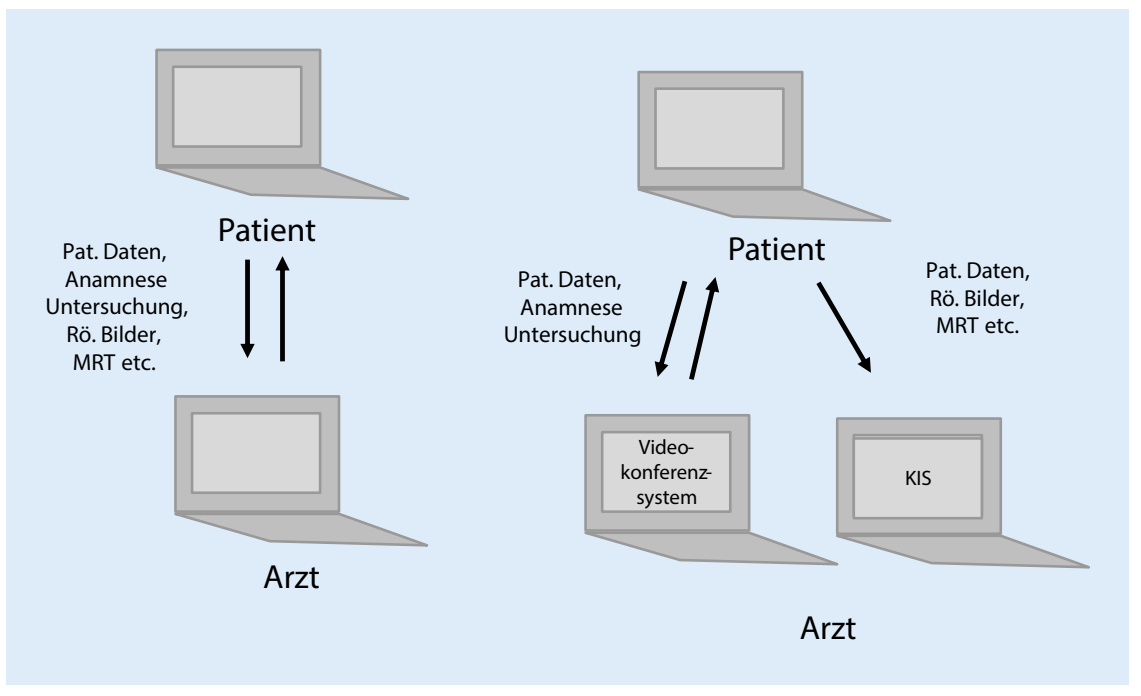

Abb. 2 \ Möglichkeiten der telemedizinischen Kommunikation. a Ein System, b zwei Systeme mit Trennung von Dokumentation und Kommunikation. KIS Krankenhausinformationssystem

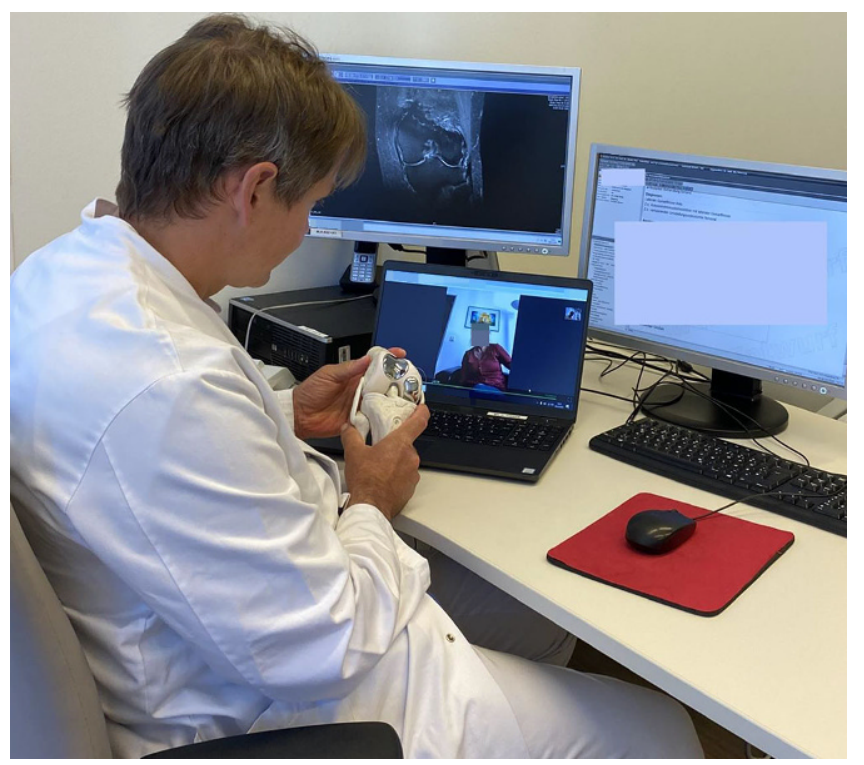

Abb. $3 \Delta$ Beispiel für eine videobasierte Sprechstunde. Hier erfolgt die Kommunikation über den Anbieter der Videosprechstunde. Die Begutachtung der radiologischen Befunde und die Dokumentation der Sprechstunde erfolgt im Krankenhausdokumentationssystem. Die Nutzung von Modellen zum Erklären von Maßnahmen kann die Effektivität wie bei der konventionellen Konsultation erhöhen

tiven Untersuchungsbefunden und Systemen zur Anleitung und Kontrolle von Rehabilitationsübungen unterschieden werden $[13,21,22,24]$. Die webbasierte ärztliche Überwachung von postoperativen Untersuchungsbefunden wurde bisher in der Knie- und Hüftendoprothetik erfolgreich eingesetzt [21, 22]. Dabei wurden die Patienten via E-Mail an Untersuchungstermine erinnert und aufgefordert, einen Fragebogen mit Gesundheitsfragen $\mathrm{zu}$ beantworten und leitung erfolgreich eingesetzt (z. B. Kommunikation Physiotherapeut/Patient) [26]. Die wissenschaftliche Datenlage ist $\mathrm{zu}$ diesem Einsatzbereich jedoch noch überschaubar. Insbesondere mit Blick auf ökonomische Aspekte liegen hier aber sicher große Möglichkeiten für die Zukunft. Schließlich kann die Ausführung von Übungen mittels moderner Technologie relativ einfach überwacht und auch korrigiert werden.

In der Nachbehandlung nach Kreuzbandrekonstruktion ist die „home-based physiotherapy" im Ausland mittlerweile etabliert $[2,17,29,30]$. Ein deutschsprachiges „Home-based-physiotherapy"-Programm ist das "Genu-Move“Programm [30]. Dabei handelt es sich jedoch nur um eine Anleitung zum eigenständigen Üben ohne telemedizinische Kontrollmöglichkeit. Eine webbasierte Version der "home-based physiotherapy" bietet das sogenannte TRAKACL-System (https://spas.cs.cf.ac.uk/ trakacl/). Dieses bietet ein individualisiertes Übungsprogramm mit Videos, Instruktionen, Gesundheitsinformationen und eine Kontaktoption mit der E-Mail eines Physiotherapeuten für zusätzliche Unterstützung. Die klinischen Erfahrungen mit diesem System waren ermutigend [13]. Die Patienten berichteten, dass das TRAK-ACL, insbesondere die Videos, ihr Selbstvertrauen und ihre Motivation bei der Rehabilitation steigerten.

\section{Sensorgestützte Diagnostik und Rehabilitation}

Große Erwartungen werden auch an den Einsatz von Sensoren zur Überwachung von Bewegungen in der Rehabilitation gestellt. Mit Sensoren können Gelenkbewegungen zuverlässig erfasst und ausgewertet werden.

Heute sind leistungsfähige Sensoren so klein, dass sie lokal am Knie angebracht werden können und auf diese Weise Bewegungen registrieren. Außerdem sind Sensoren bereits so kostengünstig erhältlich, dass ihre Anwendung in der Überwachung von Therapien und Rehabilitationen realistisch ist. Die Vernetzung mit webbasierten Anwendungen macht den 
Tab. 2 Patientencheckliste für die Videosprechstunde [35]

Legen Sie sich einen Filzstift bereit, in manchen Fällen wird es notwendig sein, auf Anweisung Ihrer Ärztin oder Ihres Arztes kleine Markierungen z. B. an der Kniescheibe vorzunehmen, um Ihre Schmerzen besser lokalisieren und einem Krankheitsbild zuordnen zu können

Tragen Sie für die videogestützte orthopädische Untersuchung der unteren Extremitäten eine kurze Hose und ziehen Sie Schuhe und Socken aus

Schaffen Sie optimale Lichtverhältnisse und vermeiden Sie Reflexion, z. B. indem Sie eine Lampe bereitstellen oder bei starker Sonneneinstrahlung die Vorhänge zuziehen

Positionieren Sie Ihre Kamera so, dass Ihre Beine vollständig sichtbar sind (in 0,6-0,9 m Höhe, z. B. auf einen Stuhl)

Setzen Sie sich frontal oder seitlich auf einen Stuhl oder die Couch

Einsatz von Sensoren auch besonders für die Telerehabilitation interessant.

Eine kürzlich publizierte Studie hat gezeigt, dass die Bewegungserfassung des Kniegelenkes in der Sagittalebene mittels zwei lokal am Knie angebrachter Sensoren ähnlich präzise war wie mit einer markergestützten Bewegungsanalyse [19]. In verschiedenen wissenschaftlichen Studien wurden lokal angebrachte Sensoren schon zur Überwachung der Rehabilitation nach Kniegelenksprothetik angewandt $[10,26,37]$.

Ein praktikables Sensorsystem ist bereits kommerziell erhältlich (Orthelligent, OPED, Valley). Dieser Sensor wird unterhalb des Kniegelenkes am Unterschenkel angebracht (• Abb. 7). Mit diesem Sensor können verschiedene funktionelle Tests (Bewegungsausmaß, Koordination, Kraft, Schnelligkeit) erfasst werden, die der Patient selbstständig durchführen kann, wenn diese vorher unter Anleitung erlernt wurden. Eine App (Orthelligent Pro App) leitet den Patienten $\mathrm{zu}$ den verschiedenen Bewegungstests an, welche anschlieBend direkt ausgewertet werden. Die Überwachung der Tests kann zusätzlich optisch durch ein von Orthelligent unabhängiges Videosystem erfolgen. Die Untersuchungsergebnisse werden in ei-
Tab. 3 Anamnesefragebogen für die Videosprechstunde

\begin{tabular}{|c|c|c|c|}
\hline Name & & & \\
\hline Vorname & & & \\
\hline Geburtsdatum & & & \\
\hline Geschlecht & & & \\
\hline Alter & & & \\
\hline Auftreten der Beschwerden & Unfall $\square$ & Kein Unfall $\square$ & \\
\hline Schmerzen & Ruhe $\square$ & Belastung $\square$ & \\
\hline Schmerzfreie Gehstrecke & $>1 \mathrm{~km} \square$ & $>500 \mathrm{~m} \square$ & \\
\hline Hilfsmittel (z. B. Gehstützen, Rollator) & $\mathrm{Ja} \square$ & Nein $\square$ & \\
\hline Schwellneigung & $\mathrm{Ja} \square$ & Nein $\square$ & \\
\hline Bewegungseinschränkung & $\mathrm{Ja} \square$ & Nein $\square$ & \\
\hline Instabilitätsgefühl & $\mathrm{Ja} \square$ & Nein $\square$ & \\
\hline Rötung/Überwärmung & $\mathrm{Ja} \square$ & Nein $\square$ & \\
\hline Stattgehabte Injektionen & $\mathrm{Ja} \square$ & Nein $\square$ & \\
\hline Stattgehabte Operationen & $\mathrm{Ja} \square$ & Nein $\square$ & \\
\hline Aktive/r Sportler/-in & Ja $\square$ & Nein $\square$ & \\
\hline Vorerkrankungen & Rheuma $\square$ & Gicht $\square$ & Sonstige $\square$ \\
\hline Medikamente & & & \\
\hline Allergien & & & \\
\hline
\end{tabular}

ne digitale Datenbank übertragen und sind so vom Arzt oder Untersucher auch online fernab des Untersuchungsortes einsehbar. Die Haupteinsatzgebiete für dieses Sensorsystem liegen in der Rehabilitation nach Knieverletzungen und Knieoperationen (z.B. „return to sport“ nach VKB(Vorderes Kreuzband)-Plastik). Ein weiterer Einsatzbereich könnte auch in der sportmedizinischen Betreuung von Leistungssportlern liegen, wenn sich diese in Trainingslagern oder auf Wettkämpfen im Ausland befinden [33]. Aber auch für die Rehabilitation nach Knieendoprothetik ist dieses System interessant.

In der klinischen Praxis ist das Orthelligent System bereits etabliert und einfach in der Anwendung. Wissenschaftliche Daten zur Validierung des Systems sind nach unserem Kenntnisstand bisher nicht publiziert.

\section{Wissenschaftliche Evidenz für den Einsatz der Telemedizin in der Orthopädie und Unfallchirurgie}

Verschiedene Autoren haben bereits vor Ausbruch der SARS-CoV-2-Pandemie die Rolle der Telemedizin in der Orthopädie und Unfallchirurgie wis- senschaftlich untersucht [7-9, 11, 21, 22, 25]. Dabei haben die meisten Studien jedoch Arzt-zu-Arzt-Konsultationen analysiert. Diese Studien stammten entweder aus der Militärmedizin oder aus dünn besiedelten Ländern [7-9, 31, 36]. - Tab. 4 fasst verschiedene wissenschaftliche Studien zur Evidenz telemedizinischer Anwendungen in der Orthopädie und Unfallchirurgie zusammen.

\section{Videosprechstunde}

Zur Bedeutung der Videosprechstunde haben wir verschiedene Studien gefunden. Hervorzuheben ist aufgrund des hochwertigen Studiendesigns eine randomisierte kontrollierte Studie aus Norwegen, deren Ergebnisse auf drei Publikationen aufgeteilt wurden [7-9].

Buvik et al. verglichen Video-Konsultationen mit Standardsprechstunden bei orthopädischen Patienten. Vierhundert Patienten wurden webbasiert randomisiert [7-9]. Von diesen wurden 199 Patienten (98\%) einer Fernkonsultation und 190 Patienten (95\%) einer Standardberatung unterzogen [7-9]. Die fachliche Bewertung (primärer Endpunkt) durch die durchführenden Ärzte war bei der Standardkonsultation zwar signifikant besser als bei Fernkonsultation (1,72 gegenüber 


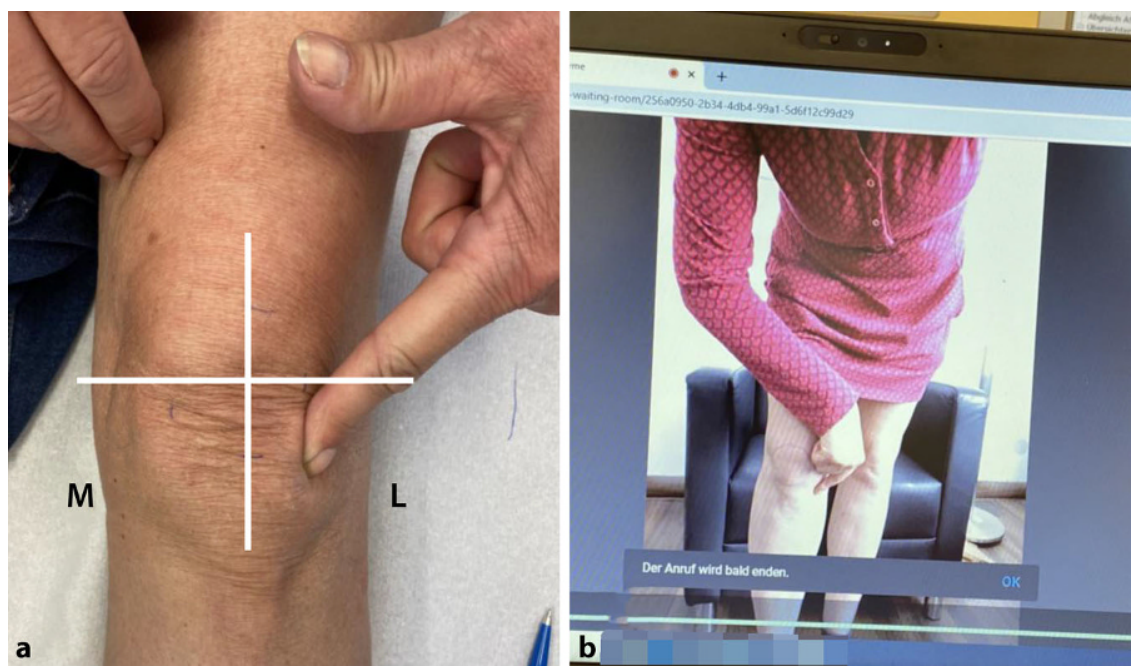

Abb. $4 \Delta$ Quadrantenmethode zur telemedizinischen Evaluation von Eigen-Palpationsbefunden in der Frontalebene. Der Patient wird aufgefordert, die Patella zirkulär zu markieren und dann mit dem Finger auf den häufigsten Schmerzpunkt zu zeigen. a Markierung der Patella und Einteilung des Kniegelenkes in 4 Quadranten. b Telemedizinische Erfassung des Palpationsbefundes in der Praxis

$1,82, p=0,0030)$; das Konfidenzintervall lag jedoch innerhalb der Nicht-Minderwertigkeitsspanne [7-9]. Auch bei weiteren sekundären Endpunkten gab es keine signifikanten Unterschiede. Die Dauer der Konsultation betrug 20,9 min in der Standardsprechstundengruppe und 20,5 min in der Telemedizingruppe [7-9]. Auch hinsichtlich der OP-Indikationen (17\% in der Standardgruppe und $11 \%$ in der Telemedizingruppe) und Komplikationen $(21 \%$ in der Standardgruppe und $17 \%$ in der Telemedizingruppe) bestanden keine signifikanten Unterschiede [7-9]. Es bestand kein Unterschied in den "patient reported outcome measures"(PROM). Der EQ-5D Index Score betrug 0,77 in der konventionellen und 0,75 in der Telemedizingruppe $(p=0,42)$ [8]. Die Kosten der Videosprechstunde waren nach 151 Patientenkonsultationen geringer als die der konventionellen Sprechstunde [9].

Die Autoren zogen die Schlussfolgerung, dass sich videounterstützte Konsultationen für orthopädische Patienten eignen [7-9]. Junge Patienten mit Knieproblemen (sporttraumatologische Diagnosen) und Schulterpatienten wurden jedoch von der Studie ausgeschlossen, da hier nach Ansicht der Autoren funktionelle Untersuchungen (Kniestabilität, Schultertests) eine große Rolle in der Diagnostik spielen [7-9].
Zwei kleinere Kohortenstudien konnten ebenfalls über positive Erfahrungen nach dem Einsatz einer Videosprechstunde berichten. Videokonferenzgestützte Untersuchungen von ambulanten orthopädischen Patienten in einer Grundversorgungseinheit brachten im Vergleich zu Konsultationen in einer Krankenhausambulanz eine vergleichbare Patientenzufriedenheit mit sich [14].

\section{Telerehabilitation}

Zum Einsatz der Telemedizin zur Überwachung der postoperativen Rehabilitation ist im Jahre 2017 ein systematisches Review publiziert worden [26]. In dieses systematische Review konnten 15 Studien mit insgesamt 1316 Teilnehmern eingeschlossen werden. Insgesamt 1316 Teilnehmer erhielten in den ausgewählten Studien eine Telerehabilitationsmaßnahme, wobei $75 \%$ aller Studien einen Knie- und Hüftersatz ausmachten. Für die telemedizinische Überwachung der Rehabilitation nach Implantation einer Knie- und Hüftprothese bestand starke und mäßige Evidenz (Empfehlungsgrad A-B). Studien zur oberen Extremität machten $25 \%$ der Studien aus, von diesen zeigte nur eine Studie einen mäßigen Evidenzgrad (Grad der Empfehlung B). Der Rest der Studien zur oberen Extremität war von schlechter methodischer
Qualität mit schwacher Evidenz (Grad der Empfehlung C).

In ein neueres systematisches Review aus dem Jahre 2018 wurden nur 4 prospektiv randomisierte Studien mit insgesamt 442 Patienten eingeschlossen [18]. In diesem Review erreichten Patienten nach Telerehabilitation eine signifikant bessere Extension und Quadrizepskraft als nach Präsenzrehabilitation bei vergleichbarem Schmerzniveau [18]. Auch der Western Ontario McMaster Universities Osteoarthritis Score war nach Telerehabilitation höher [18].

Hinsichtlich des Einsatzes des TRAKACL-Systems in der Rehabilitation nach Rekonstruktion des vorderen Kreuzbandes wurde bisher eine Studie publiziert [13]. In diese konnten 24 Patienten eingeschlossen werden. In dieser Studie erwies sich TRAK-ACL als akzeptable Methode zur Durchführung einer Rehabilitation nach VKB-Plastik. Die Patienten berichteten, dass TRAK, insbesondere die Videos, ihr Selbstvertrauen und ihre Motivation bei der Rehabilitation steigerten.

\section{Fazit}

Erste telemedizinische Ansätze in der Orthopädie und Unfallchirurgie sind ermutigend. Gerade jetzt zu Zeiten der SARSCoV-2-Pandemie mit der Notwendigkeit von Kontaktbeschränkungen wächst auch in einem dicht besiedelten Land wie Deutschland das Interesse an diesen Anwendungen. Insbesondere am Kniegelenk lässt sich mit dem Einsatz von Videotechnik die telemedizinische Anamnese kostengünstig durch einfache Untersuchungsbefunde (Inspektion, Eigenpalpation, Beweglichkeit, einfache Tests) ergänzen. Nachteil ist, dass funktionelle Tests (z.B. Kniestabilität) nicht durchführbar sind und auch die Inspektion abhängig von den technischen Voraussetzungen ist. Als Limitationen der telemedizinischen Behandlung auf Patientenseite können darüber hinaus kognitive Einschränkungen (u. a. Erkrankungen aus dem dementiellen Formenkreis) mit Problemen bei der aktiven Beteiligung an den geforderten Untersuchungsgängen genannt werden. Weiterhin bestehen für die Durchführung von videobasierten Behandlungsformaten Anforderun- 


\section{Übersichten}
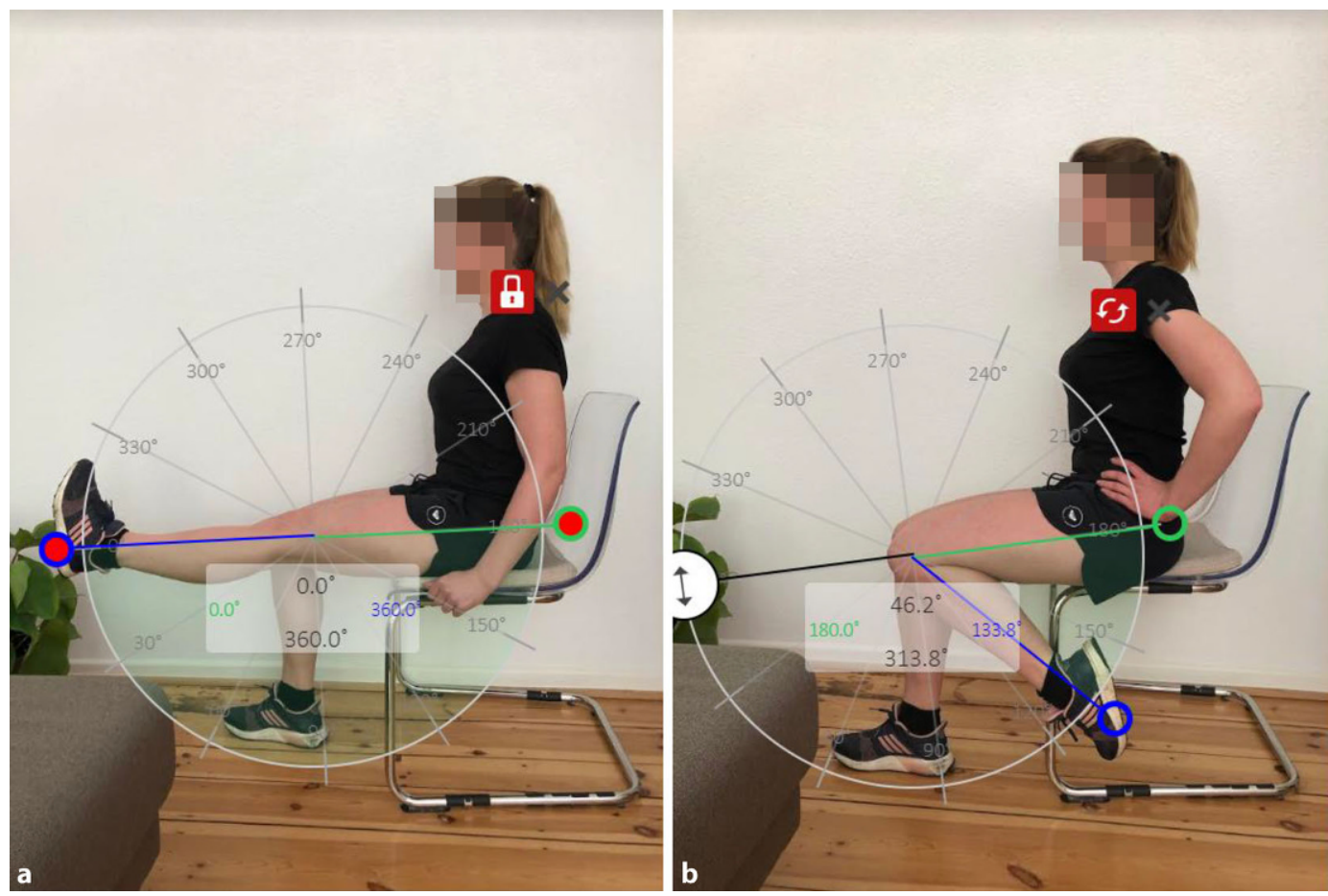

Abb. $5 \triangleleft$ Beurteilung der Beweglichkeit im Sitzen unter Zuhilfenahme eines webbasierten Goniometers (Protractor; Ben Burlingham [32])

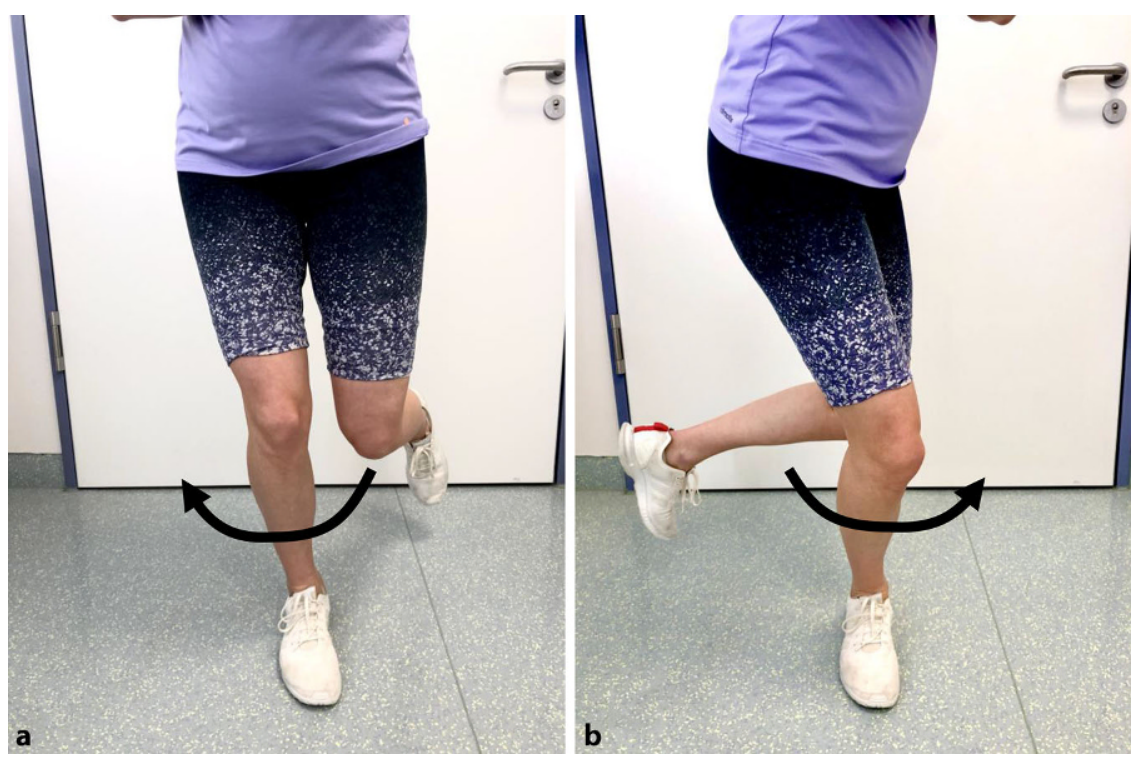

Abb. 6 A Ein telemedizinisch durchführbarer Meniskustest ist der Thessaly-Test, bei dem der Patient auf dem betroffenen Bein steht und den Körper nach $\mathbf{a}$ außen und $\mathbf{b}$ innen dreht. Als positiver Befund gelten Schmerzen und mechanische Missempfindungen über der Seite des betroffenen Meniskus

gen an die verfügbare Internetinfrastruktur, wobei nach Angaben des Bundesministeriums für Verkehr und digitale Infrastruktur $201990 \%$ der Bundesbürger über Breitbandinternet ( $\geq 50 \mathrm{Mbit} / \mathrm{s})$ verfügten [6]. Bisherige wissenschaftliche Daten zur Evidenz von Videokonsultationen sind jedoch ermutigend. Hier besteht allerdings noch großer Handlungsbedarf, um insbesondere videogestützte
Untersuchungsmethoden weiter zu validieren. $\mathrm{Zu}$ den Vorteilen telemedizinischer Anwendungen können die Vermeidung langer Anfahrtswege, die bessere Anbindung ländlicher Regionen an spezialisierte medizinische Versorgung sowie die resultierende Kosteneffizienz genannt werden. Auch in der Rehabilitation nach Knieverletzungen und Knieoperationen werden telemedizinische Appli-

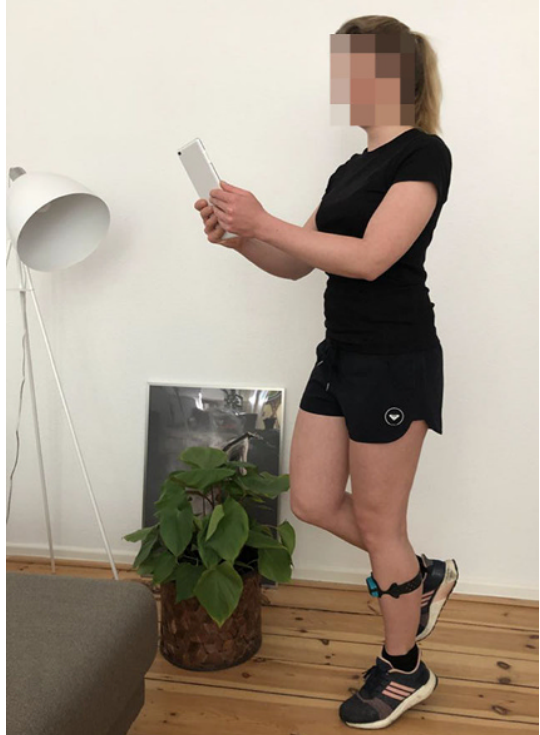

Abb. $7 \triangle$ Anwendung eines Bewegungssensors (Orthelligent, OPED, Valley) zur Anwendung in der postoperativen Rehabilitation. Mit diesem Sensor können verschiedene, für die Rehabilitation relevante Parameter erfasst werden (Bewegungsausmaß, Koordination, Kraft, Schnelligkeit). Der Einsatz dieses Systems ist auch im Bereich der Telemedizin möglich, da die gewonnenen Daten in einer zentralen Datenbank erfasst werden und dem Untersucher webbasiert übermittelt werden können 


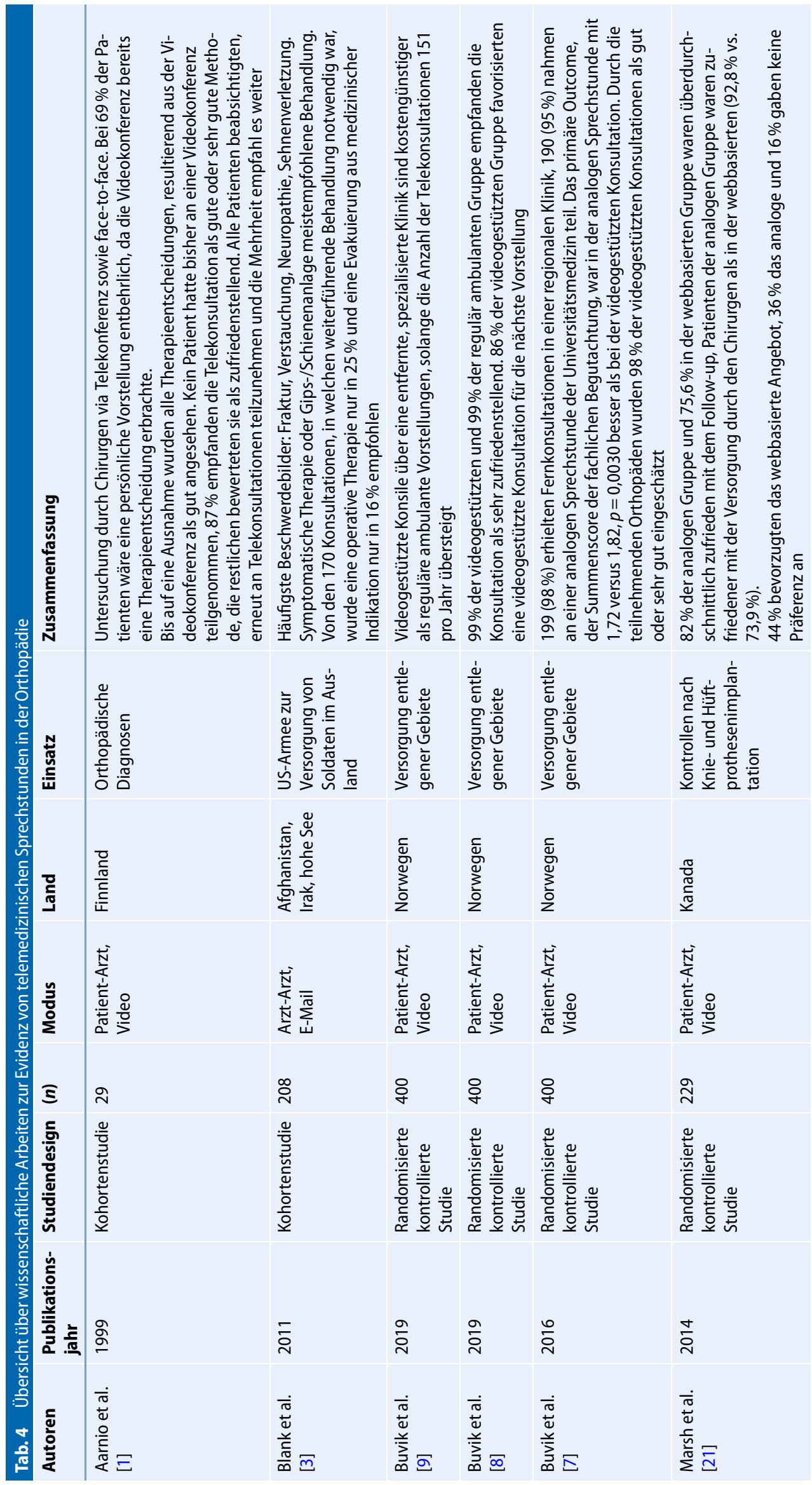




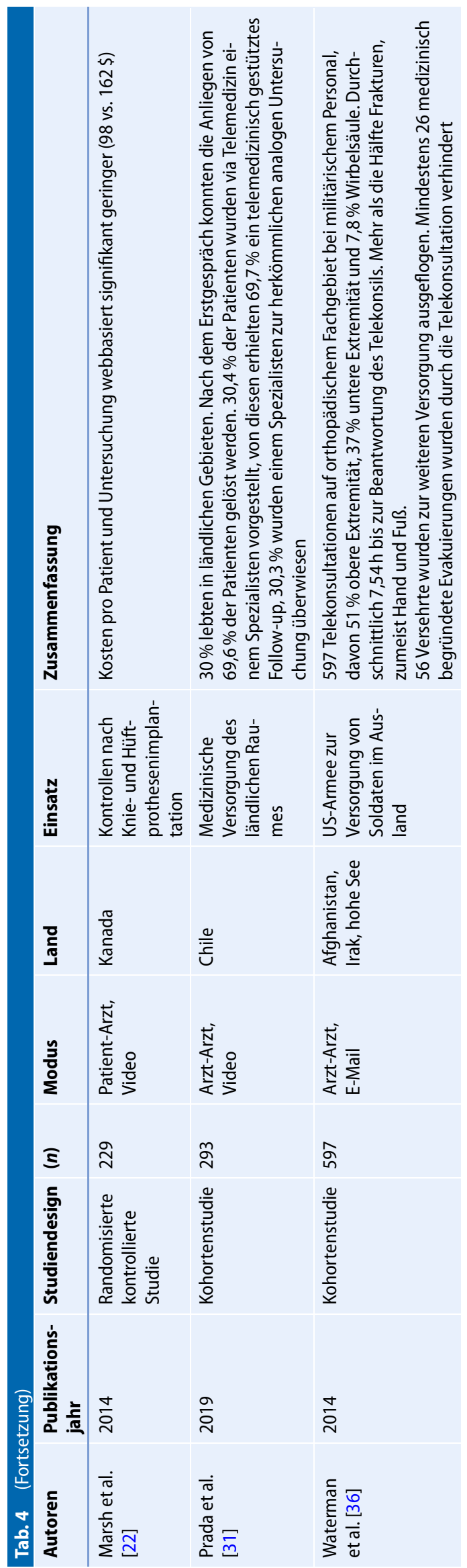

kationen bereits eingesetzt und bieten in Zeiten der SARS-CoV-2-Pandemie eine Möglichkeit zur kontaktlosen Therapieüberwachung aus der Ferne. Diese Technologien sollten weiter ausgebaut werden, da sie auch nach der Coronakrise Möglichkeiten z. B. in der Betreuung von Leistungs- und Profisportlern oder in der Betreuung von internationalen Patienten bieten.

\section{Korrespondenzadresse}

Prof. Dr. med. Wolf Petersen

Sportklinik Berlin und Klinik für Orthopädie und Unfallchirurgie, Martin Luther Krankenhaus, Berlin Grunewald

Caspar-Theyß-Straße 27-31, 14193 Berlin, Deutschland

wolf.petersen@jsd.de

\section{Einhaltung ethischer Richtlinien}

Interessenkonflikt. L. Backhaus, S. Bierke, K. Karpinskiund M. Häner geben an, dass kein Interessenkonflikt besteht. WolfPetersen gibtan, Beratungshonorare von den Firmen Karl Storz und OttoBock Health Care sowie Vortragshonorare der Firmen Plasmaconcept sowie Arthrex erhalten zu haben.

Für diesen Beitrag wurden von den Autoren keine Studien an Menschen oder Tieren durchgeführt. Für die aufgeführten Studien gelten die jeweils dort angegebenen ethischen Richtlinien.

\section{Literatur}

1. Aarnio P, Lamminen H, Lepistö J, Alho A (1999) A prospective study of teleconferencing for orthopaedic consultations. J Telemed Telecare 5(1):62-66

2. Achtnich A, Akoto R, Petersen W (2019) Handlungsalgorithmus: Wiederkehr zum Sport nach Rekonstruktion des vorderen Kreuzbandes. Knie J. https://doi.org/10.1007/s43205-019-00011-5

3. Blank E, Lappan C, Belmont PJ Jr, Machen MS, Ficke J, Pope R, Owens BD (2011) Early analysis oft he United States Army's telemedicine orthopaedic consultation program. J Surg Orthop Adv 20(1):50-55

4. Blonna D, Zarkadas PC, Fitzsimmons JS, O'Driscoll SW (2012) Validation of a photography-based goniometry method for measuring joint range of motion. J Shoulder Elbow Surg 21(1):29-35

5. Boissy $P$, Tousignant $M$, Moffet $H$, Nadeau $S$, Brière $S$, Mérette $C$, Corriveau $H$, Marquis $F$, Cabana F, Ranger P, Belzile ÉL, Dimentberg R (2016) Conditions of use, reliability, and quality of audio/video-mediated communications during in-home rehabilitation teletreatment for postknee arthroplasty. Telemed J E Health 22(8):637-649

6. Bundesministerium für Verkehr und digitale Infrastruktur (2018) Von der digitalen in die Gigabit-Gesellschaft. https://www.bmvi.de/DE/ 
Themen/Digitales/Breitbandausbau/Breitbandkompakt/breitband-kompakt.html. Zugegriffen: 3. Mai 2020

7. Buvik A, Bugge $E$, Knutsen G, Småbrekke A, Wilsgaard T (2016) Quality of care for remote orthopaedic consultations using telemedicine: a randomised controlled trial. BMC Health Serv Res 16:483. https://doi.org/10.1186/s12913-0161717-7

8. Buvik A, Bugge $E$, Knutsen $G$, Småbrekke $A$, Wilsgaard T (2019) Patient reported outcomes with remote orthopaedic consultations by telemedicine: A randomised controlled trial. J Telemed Telecare 25(8):451-459. https://doi.org/10.1177/ $1357633 \times 1878392$

9. Buvik A, Bergmo TS, Bugge E, Smaabrekke A, Wilsgaard T, Olsen JA (2019) Cost-effectiveness of telemedicine in remote orthopedic consultations: Randomized controlled trial. J Med Internet Res 21(2):e11330.https://doi.org/10.2196/11330

10. Calliess T, Bocklage R, Karkosch R, Marschollek M, Windhagen H, Schulze M (2014) Clinical evaluation of a mobile sensor-based gait analysis method for outcome measurement after knee arthroplasty. Sensors (Basel) 14(9):15953-15964

11. DeldarK, BahaadinbeigyK, Tara SM (2016) Teleconsultation and clinical decision making: Asystematic review. Acta Inform Med 24(4):286-292

12. Dent PA Jr, Wilke B, Terkonda S, Luther I, Shi GG (2020) Validation of teleconference-based goniometry for measuring elbow joint range of motion. Cureus 12(2):e6925

13. Dunphy E, Hamilton FL, Spasić I, Button K (2017) Acceptability of a digital health intervention alongside physiotherapy to support patients following anterior cruciate ligament reconstruction. BMC Musculoskelet Disord 18(1):471-421. https:// doi.org/10.1186/s12891-017-1846-0

14. Haukipuro K, Ohinmaa A, Winblad I, Linden T, Vuolio S (2000) The feasibility of telemedicine for orthopaedic outpatient clinics-a randomized controlled trial. J Telemed Telecare 6(4):193-198

15. health innovation hub (2020) Corona-Datenspende: Ein Fieberthermometer für Deutschland. https://hih-2025.de/corona/. Zugegriffen: 5. Mai 2020

16. Hirschmann MT, Hart A, Henckel J, Sadoghi $P$, Seil R, Mouton C (2020) COVID-19 coronavirus: recommended personal protective equipment for the orthopaedic and trauma surgeon. Knee Surg Sports Traumatol Arthrosc. https://doi.org/ 10.1007/s00167-020-06022-4

17. Hohmann E, Tetsworth K, Bryant A (2011) Physiotherapy-guided versus home-based, unsupervised rehabilitation in isolated anteriorcruciate injuries following surgical reconstruction. Knee Surg Sports Traumatol Arthrosc 19(7):1158-1167

18. Jiang S, Xiang J, Gao X, Guo K, Liu B (2018) The comparison of telerehabilitation and face-to-face rehabilitation after total knee arthroplasty: $A$ systematic review and meta-analysis. J Telemed Telecare 24(4):257-262

19. Kayaalp ME, Agres AN, Reichmann J, Bashkuev M, Duda GN, Becker R (2019) Validation of a novel device for the knee monitoring of orthopaedic patients. Sensors (Basel) 19(23):27

20. Karachalios T, Hantes M, Zibis AH, Zachos V, Karantanas AH, Malizos KN (2005) Diagnostic accuracy of a new clinical test (the Thessaly test) for early detection of meniscal tears. J Bone Joint Surg Am 87(5):955-962

21. Marsh J, Bryant D, MacDonald SJ, Naudie D, Remtulla A, McCalden R, Howard J, Bourne R, McAuley J (2014) Are patients satisfied with a web- based followup after total joint arthroplasty? Clin Orthop Relat Res 472(6):1972-1981

22. Marsh J, Hoch JS, BryantD, MacDonald SJ, NaudieD, McCalden R, Howard J, Bourne R, McAuley J (2014) Economicevaluation of web-based compared with in-person follow-up after total joint arthroplasty. JBone Joint Surg Am 96(22):1910-1916

23. Meislin MA, Wagner ER, Shin AY (2016) A comparison of elbow range of motion measurements: Smartphone-based digital photography versus goniometric measurements. J Hand Surg Am 41(4):510-515.e1

24. Naeemabadi $M$, Dinesen $B$, Andersen OK, Madsen NK, Simonsen OH, Hansen J (2019) Developing a telerehabilitation programme for postoperative recovery from knee surgery; specifications and requirements. BMJ Health Care Inform 26(1):e22

25. Ohinmaa A, Vuolio S, Haukipuro K, Winblad I (2002) A cost-minimization analysis of orthopaedic consultations using videoconferencing in comparison with conventional consulting. J Telemed Telecare 8(5):283-289

26. Pastora-Bernal JM, Martín-Valero R, BarónLópez FJ, Estebanez-Pérez MJ (2017) Evidence of benefit of telerehabitation after orthopedic surgery: a systematic review. J Med Internet Res 19(4):e142. https://doi.org/10.2196/jmir.6836

27. Petersen W, Rembitzki I, Liebau C (2017) Patellofemoral pain in athletes. Open Access J Sports Med 8:143-154. https://doi.org/10.2147/OAJSM. S133406

28. Petersen W, Bierke S, Karpinski K, Häner M (2020) Coronavirus-Pandemie und ihre Auswirkungen auf Orthopädie und Unfallchirurgie: Operationen, Risiken und Prävention? Knie J. https://doi.org/10 1007/s43205-020-00052-1

29. Petersen W (im Druck) Phasen und kriterienbasierte Rehabilitation nach Rekonstruktion des vorderen Kreuzbandes. Thieme Refresher 2020

30. Petersen W Genu move programm. https:// media.ottobock.com/_web-site/orthotics/genuarexa-50k18/files_2018/2892204_genu_arexa_ ubungen_genu_move_ottobock.pdf. Zugegriffen: 3 . Mai 2020

31. Prada C, Izquierdo N, Traipe R, Figueroa $C$ (2019) Results of a new Telemedicine strategy in traumatology and orthopedics. Telemed JEHealth. https://doi.org/10.1089/tmj.2019.0090

32. ben.burlingham (2020) Protractor. https:// chrome.google.com/webstore/detail/protractor/ kpjldaeddnfokhmgdlmpdlecmobaonnj.Zugegriffen: 5 . Mai 2020

33. Rigamonti L, Albrecht UV, Lutter C, Tempel M, Wolfarth B, Back DA (2020) Working group Digitalisation. Potentials of digitalization in sports medicine: a narrative review. Curr Sports Med Rep 19(4):157-163

34. Robert Koch-Institut (2020) SARS-CoV-2 Steckbrief zur Coronavirus-Krankheit-2019 (COVID19). https://www.rki.de/DE/Content/InfAZ/ N/Neuartiges_Coronavirus/Steckbrief.html\# doc13776792bodyText2.Zugegriffen:5. Apr. 2020

35. Tanaka M, Oh LS, Scott M, Berkson E (2020) Telemedicine in the era of COVID-19. J Bone Joint Surg Am. https://doi.org/10.2106/JBJS.20.00609

36. Waterman BR, Laughlin MD, Belmont PJ Jr, Schoenfeld AJ, Pallis MP (2014) Enhanced casualty care from a global military orthopaedic teleconsultation program. Injury 45(11):1736-1740

37. Zhang HH, Yan SH, Fang C, Guo XY, Zhang K (2016) Clinical evaluation and gait characteristics before and after total knee arthroplasty based on a portablegait analyzer. OrthopSurg 8(3):360-366. https://doi.org/10.1111/os.12270

38. Zhao JZ, Blazar PE, Mora AN, Earp BE (2019) Range of motion measurements oft the fingers via smartphone photography. Hand (N Y) 28:1558944718820955 